\title{
O MÉTODO MISTO PARA VALORAÇÃO DE PAISAGENS COSTEIRAS
}

\author{
ANDREACCI, F.'; MARENZI, R. C. ${ }^{2}$ \\ Universidade do Vale do Itajaí ${ }^{1}{ }^{2}$ \\ *Corresponding author: fandreacci@gmail.com
}

\begin{abstract}
Andreacci, F.; Marenzi, R. C. 2017. O método misto para valoração de paisagens costeiras. Braz. J. Aquat. Sci. Technol. 21(2). eISSN. DOI: 12428/bjast.v21n2. Landscape is a non-renewable resource, which is understood as the expression of the product of spatial and temporal interaction of the individual with the environment. Valuation of the quality of this resource is an important tool for planning and managing the territory, once it is related with the positive and negative effects that landscape components evoke in individuals. This work show in detail the mixed method for landscape valuation and discusses its application in coastal environments.
\end{abstract}

\section{INTRODUÇÃO}

A paisagem pode ser considerada como um recurso não renovável e não consumido diretamente pelas pessoas, mas que pode sofrer degradação irreversível por elas (Bolós et al., 1992). Esse recurso é de difícil conceituação, uma vez que é entendido como a expressão do produto de interação espacial e temporal do indivíduo com o meio (UICN, 1984). Portanto, a paisagem é um patrimônio ambiental que compreende valores naturais e culturais, sendo um recurso representativo da fisionomia de um espaço que reflete sua natureza e história (Ortega, 1988).

Paisagens tranquilas e de beleza cênica, históricas e naturais são frequentemente procuradas pelas pessoas com o intuito de encontrar lazer, relaxamento e saúde mental. Assim, a paisagem também deve ser estudada e protegida em função de seu desempenho na satisfação das necessidades humanas elementares de bem-estar e auto realização, uma vez que cultura e natureza estão interligadas (Forman \& Gordon, 1986).

No território brasileiro, em decorrência de um processo de ocupação que foi implantado próximo à costa, contando com os primeiros e mais estáveis pontos de povoamento, a paisagem litorânea concorre com a expansão da malha urbana, onde concentrase a maior parte da população do país (IBGE, 2012). Nesse contexto, o processo de produção do espaço costeiro deve ser questionado pelas próprias contradições de sua organização, onde cada loteamento tende a reproduzir, na praia, o ideário do subúrbio urbano com residências isoladas ou, em outros pontos de elevado valor, constituindo situações de alta densidade de construções verticalizadas. Assim, as pessoas que buscam sair da cidade e das suas formas de organização espacial se deparam com sua própria réplica à beira-mar. Portanto, de acordo com Pelegrino (1989), cada lugar na costa deve ser avaliado pelos seus valores cênicos e ambientais, pela disponibili- dade de recursos existentes, suas possibilidades de esgotamento, reposição e transformação.

A partir do exposto, o intuito deste trabalho é descrever uma metodologia mista aplicada na valoração da paisagem utilizando elementos de fácil mensuração e que quando aplicada, forneça resultados que possam ser utilizados como ferramentas de gestão do território. Ainda, são apresentados os resultados da aplicação do método para ambientes costeiros e discutimos brevemente sua importância.

\section{Método misto para valoração da paisagem}

Diversos são os métodos utilizados para valoração da paisagem, diretos, indiretos ou mistos (Panagopoulos, 2009; Mayoh et al., 2015; Loures et al., 2015). Se por um lado os métodos diretos exprimem um nível elevado de subjetividade, os indiretos se baseiam no juízo de valores segundo critérios estabelecidos por especialistas (Bolós, 1992; Loures et al., 2015). Por sua vez, os métodos mistos tentam combinar as duas tendências, gerando uma aproximação mais eficaz da qualidade da paisagem. Essa técnica pode ser resumida nos seguintes passos: a), divisão do território em unidades ambientais; b) amostragem da área através de substitutos da paisagem; c), identificação dos componentes ou variáveis ambientais dos substitutos; d), mensuração de cada componente nos substitutos; e), valoração de cada um dos substitutos das paisagens por um método direto; f) realização de regressões múltiplas a partir dos dados obtidos e h), escolha do melhor modelo de regressão para determinar os pesos dos componentes (Marenzi, 2006). Ainda, existem diversas modificações do método, com o uso de métodos multivariados. Divisão do território em unidades de paisagem.

O território em avaliação pode ser dividido em função de um componente dominante da paisagem, como topografia, vegetação, atividade antrópica, ou a fisionomia em geral. Essa divisão tende a ser maior quanto menor for a escala de trabalho e permite um 
melhor controle da análise.

\section{Amostragem por substitutos da paisagem}

Fotografias são bons substitutos da paisagem, pois permitem descrições das preferências da paisagem que elas representam, apresentam praticidade e padronização da análise. Contudo, as fotografias também reduzem os limites dos objetos, redimensiona a paisagem tridimensional em um conjunto bidimensional de formas geométricas e pode induzir o julgamento do observador, uma vez que este encontra-se, geralmente, em um local distante do ponto observado. Mesmo assim, a utilização de fotografias tem se demonstrado eficaz na valoração de paisagens (Kent, 1993; Marenzi, 2006; Kim \& Kang 2008; Felix et al. 2016).

\section{Componentes ambientais dos substitutos, identificação e mensuração}

As fotografias que representam paisagens podem ser decompostas em diversas subunidades como: céu, água, solo exposto, pastagem, areia, pessoas, elemento humano, edificações, etc. A quantidade de componentes identificados depende do nível de detalhamento desejado pelo pesquisador, podendo ser as fotografias divididas em quadriculas maiores ou menores mediante a escala adotada. Dependendo da quantidade de subunidades identificadas e utilizadas em um trabalho, maior deverá ser o número de fotografias valoradas. Isso se dá em decorrência da utilização da técnica de regressão múltipla mais adiante no método. Nesse caso, os componentes identificados serão considerados como preditores, ou variáveis independentes, da qualidade visual da fotografia (Figura 1).

Existem vários métodos para determinar o tamanho mínimo da amostra para realizar uma regressão múltipla (Van Voorhis \& Morgan, 2007). Contudo, como regra geral, não se deve utilizar uma amostra inferior a 50 , podendo este número ser aumentado mediante ao número de variáveis independes. De acordo com Harris (1985), equações de regressão com um número de preditores igual ou maior a seis devem utilizar no mínimo dez amostras por preditor, sendo 30 amostras o número ideal para detectar até mesmo efeitos de pequeno tamanho.

\section{Valoração das fotografias}

Definidos os componentes ambientais das fotografias, e, consequentemente, o número de fotografias suficientes para estimar esses componentes, procurase efetuar a valoração da qualidade da paisagem expressa na fotografia. Nessa etapa, as paisagens expressas nas fotografias são valoradas através do método direto por um agente. $\mathrm{O}$ agente poderá ser $\mathrm{O}$ público em geral, grupos representativos da sociedade ou profissionais paisagistas, podendo ser subdivido por classes de subjetividade em: admitida, controlada, compartilhada e representativa. O pesquisador ainda poderá coletar outros dados a respeito do agente, quanto a sua idade, escolaridade, ocupação, local de moradia, etc, permitindo uma futura divisão em grupos homogêneos e, ou, outras inferências. A valoração das fotografias deve se dar em classes de qualidade, permitindo assim obter um parâmetro de avaliação. Como sugestão, pode-se adotar a classe de pontuação de 1 a 5 para a qualidade ambiental da imagem, onde: 1 - muito baixa (péssima), 2 - baixa (ruim), 3 - média (regular), 4 - alta (boa) e 5 - muito alta (ótima) (Orea, 1976; Jordana, 1992).

Figura 1. Identificação e mensuração dos componentes ambientais da fotografia. A, fotografia original, subdivida em quadrículas. $B$, identificação dos componentes predominantes no interior da quadrícula. C, legenda.

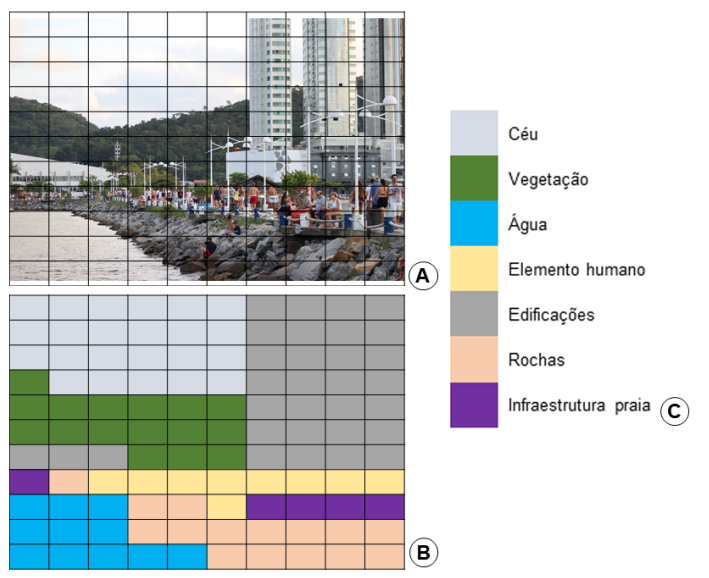

A suficiência amostral, quantidade de valorações de uma fotografia, pode ser estimada a partir de uma amostra piloto, onde com os dados da variância da média da pontuação, aplica-se a seguinte fórmula, de acordo com Steel \& Torrie (1960):

$$
n=\frac{\sigma^{2} * t^{2}}{\varepsilon^{2}}
$$

Onde: $n$, tamanho da amostra; $\sigma^{2}$, variância da fotografia; $t^{2}$, valor tabelado de acordo com os graus de liberdade e o nível de confiança desejável; $\varepsilon^{2}$, erro admissível da média desejado.

Dependendo do valor obtido, a amostra piloto pode ser considerada suficiente, caso contrário, novas valorações deverão ser tomadas a fim de se obter uma boa amostragem para cada fotografia.

\section{Regressões múltiplas a partir dos dados obtidos \\ De posse dos dados da mensuração dos com- ponentes ambientais e da valoração média de cada}


fotografia, é ajustado um modelo de regressão linear múltiplo admitindo como variável dependente $(Y)$, a média da valoração da fotografia e como variáveis independentes $\left(x_{1} x_{2} \ldots x_{p}\right)$ a quantificação dos componentes ambientais. O modelo é expresso pela seguinte fórmula:

$$
Y=\beta_{0}+\beta_{1} x_{1}+\beta_{2} x_{2}+\cdots+\beta_{p} x_{p}+\varepsilon
$$

Onde: $Y$, variável dependente; $\beta_{0}$, coeficiente linear da reta ou constante da equação de regressão; $\beta_{1} \beta_{2} \cdots \beta_{p}$ coeficientes angulares da reta ou coeficientes parciais de regressão. $x_{1} x_{2} \ldots x_{p}$ variáveis dependentes; $\varepsilon$, erro ou resíduo; indica os resíduos decorrentes de outros fatores que afetem a variável dependente, além dos considerados no modelo adotado ou por erros na mensuração.

Atualmente existem ferramentas que permitem automatizar a escolha das variáveis independentes (componentes ambientais) de maneira a selecionar o melhor modelo através de uma triagem exaustiva para frente, para trás, ou por substituição sequencial das variáveis. Essa técnica, conhecida como Stepwise (passo-a-passo), baseia-se na importância da variável definida em termos de uma medida de significância estatística do coeficiente associado à variável para o modelo. Ainda, é importante notar que a robustez do modelo de regressão linear múltipla assume uma relação linear entre a variável independente e as dependentes; requer que os resíduos da análise (erros entre os valores observados e preditos) sejam normalmente distribuídos; que não haja multicolinearidade entre as variáveis dependentes, ou seja, que elas não sejam altamente correlacionadas; e homocedasticidade, variância constante dos erros, entre outros (Allison, 1999).

De posse do melhor modelo, pode-se utilizálo para predizer a valoração das fotografias, sem o uso dos valoradores (agentes). Com isso, e levando em consideração as limitações de qualquer modelo, pode-se aplicá-lo à totalidade do território estudado para valorar outras paisagens, ou ainda em territórios semelhantes, garantindo a utilização do método como uma ferramenta de gestão e planejamento do território.

\section{Aplicações do método na análise de paisagens costeiras}

O método misto é uma técnica de valoração da paisagem de baixo custo e que pode complementar informações pertinentes à ecologia da paisagem com fins de planejamento e zoneamento de uso do solo. Em ambientes costeiros, esse método aplicado por Marenzi (1996) no litoral norte de Santa Catarina destacou o componente ambiental água como principal componente positivo na qualidade paisagística e o solo exposto como principal componente negativo.
Kim \& Kang (2008), em uma versão modificada do método aplicadas em praias da Coreia do Sul, verificaram que os agentes valoradores preferem praias com um aspecto harmonioso, que denote beleza e excitação, além de segurança. Já as análises de Felix et al. (2016) realizadas no sul do Brasil e do Uruguai, apontam que praias mais conservadas, compostas por elementos naturais mais homogêneos, são melhor avaliadas quanto a qualidade visual em detrimento de praias mais artificializadas e de maior heterogeneidade. Contudo, os autores perceberam que a presença de serviços e infraestrutura adequada nas praias urbanizadas aumenta significativamente sua valoração, denotando a importância dessas estruturas para a população.

Nesse sentido, pensando no potencial turístico dos ambientes costeiros, torna-se fundamental conhecer a qualidade visual da paisagem, uma vez que este conceito relaciona-se com os efeitos positivos ou negativos que os seus componentes da paisagem evocam nos indivíduos. Assim, a análise da qualidade da paisagem desses ambientes é uma ferramenta importante para ser utilizada no planejamento e gestão do território.

\section{REFERÊNCIAS}

Allison, P. D. 1999. Multiple regression: a primer. Pine Forge Press, California. 220p.

Bolós, M. 1992. Manual de ciência del paisage: teoria, métodos y aplicaciones. Massson, Barcelona. $193 \mathrm{p}$.

Felix, G.; Marenzi, R. C.; Polette, M. \& Netto, S. A. 2016. Landscape Visual Quality and Meiofauna Biodiversity on Sandy Beaches. Environmental Management 58(4): 682-693.

Forman, R. T. T. \& Godron, M. 1986. Landscape ecology. J.Wiley, USA. 640 p. Harris, R. J. 1985. A primer of multivariate statistics. Academic Press, New York. 576 p.

Instituto Brasileiro de Geografia e Estatística (IBGE). 2012. Censo Brasileiro de 2010. IBGE, Rio de Janeiro.

Jordana, J. C. C. 1993. Curso de Introducción al Paisage: Metodologias de Valoración. Universidade Federal do Paraná / Universidad de Cantábria, 60 p. (apostila).

Kent, R. L. Determining scenic quality along highways, a cognitive approach. 1993. Landscape and Urban Planning 27: 29-45.

Kim, N. H. \& Kang, H. K. 2008. The aesthetic evaluation of coastal landscape. Journal of Civil Engineering 13(2): 65-74.

Loures, L.; Loures, A.; Nunes, J. \& Panagopoulos, T. 2015. Landscape Valuation of Environmental 
Amenities throughout the Application of Direct and Indirect Methods. Sustainability 7: 794-810.

Marenzi, R. C. 1996. Estudo da Valoração da Paisagem e Preferências Paisagísticas no Município da Penha - SC. Dissertação de Mestrado. Universidade Federal do Paraná. 119 p.

Mayoh, J. \& Onwuegbuzie, A.J. 2015. Toward a conceptualization of mixed methods phenomenological research. J. Mixed Methods Res. 9: 91-107.

Orea, D G. 1976. El medio físico y la planifícación CIFCA, Madrid, $144 \mathrm{p}$.

Ortega, N. 1988. Paisaje y cultura. In: De Pisón, E, M. Paisaje y medio ambiente. Valladolid: Secretariado de Publicaciones e Intercambio Científico de la Universidad de Valladolid. 137-146pp.

Panagopoulos, T. 2009. Linking forestry, sustainability and aesthetics. Ecol. Econ. 68: 2485-2489.

Pedraza, J. 1986. Paisajes geológicos. El ejemplo de la Sierra de Guadarrama en su vertiente meridional. In: Ramos, A (coord). Curso Monográfico sobre Restauración del Paisaje. Problemas, bases científicas y técnicas de recuperación. Madrid: Fundación Conde del Valle de Salazar. 47-68pp.

Pellegrino, P. R. M. 1989. A paisagem possível. Paisagem e Ambiente 3: 67-75

Steel, R. \& Torrie, J. 1960. Principles and procedures of statistics: with special reference to the biological sciences. Mcgraw-Hill, New York, Toronto, London. $481 \mathrm{p}$.

União Internacional Para Conservação da Natureza - UICN. 1984. Estratégia mundial para a conservação: a conservação dos recursos vivos para um desenvolvimento sustentado. São Paulo: CESP.

Van Voorhis, C. R. W \& Morgan, B. L. 2007. Understanding Power and Rules of Thumb for Determining Sample Sizes. Tutorials in Quantitative Methods for Psychology 3(2): 43-50.

Submetido: Março/18

Revisado: Abril/18

Aceito: Julho/18

Publicado: 15 de Agosto/18 